\title{
THE ANALYSIS OF FERROELECTRIC TYPE INSTABILITIES IN THE TWO-SUBLATTICE MODEL OF HIGH TEMPERATURE SUPERCONDUCTING SYSTEMS
}

\author{
O.D.DANYLIV, I.V.STASYUK \\ Institute for Condensed Matter Physics \\ Ukrainian National Academy of Sciences \\ 1 Svientsitskii St., 290011 Lviv-11, Ukraine
}

Received May 6, 1996

\begin{abstract}
The two-sublattice pseudospin model of Mitsui type supplemented by the interaction with electron subsystem is considered. Such a model can be used for the description of dielectric properties of $\mathrm{YBaCuO}$-type superconductors along $c$-axis (the pseudospins represent anharmonic motions of apical oxygen 04). The behaviour of the the transverse dielectric susceptibility $\chi_{\perp}$ is considered in the noninteracting clusters approximation. Possibility of different temperature dependencies of $\chi_{\perp}$ is established.

The free energy of the model is evaluated in the mean field approximation. It is established that the long-range interaction between pseudospins can lead to symmetry breaking and to appearance of the ferroelectric component of pseudospin ordering. The phase transitions into the state with decompensated dipole moments can be both of second and first order. The corresponding $\left(T_{c}, h\right)$ diagrams ( $h$ is the asymmetry parameter of the field acting on pseudospins) at different values of long-range interaction constants in the regimes of fixed values of electron concentration $(n=$ const $)$ or chemical potential ( $\mu=$ const $)$ are obtained. The anomalies of susceptibility $\chi_{\perp}$ in the vicinity of phase transitions points are investigated.
\end{abstract}

\section{Introduction}

Since the discovery of high temperature superconductivity, extensive studies of theoretical models have been carried out to describe the behaviour of high temperature superconductors (HTSC). The Hubbard model was one of the first models to be proposed. It takes into account the strong correlation of electrons on the sites in the superconducting planes (such planes with hole conductivity exist in all HTSC). The analysis of EXAFS spectra and Raman spectra for $\mathrm{YBaCuO}$ [1-3] manifests in the existence of a strong anharmonic double potential well of apex oxygen $\mathrm{O} 4$. This conclusion agrees with experiments on the diffraction of X-rays [4].

In order to describe anharmonic vibrations in the case of a local potential with two minima separated by a high barrier, the pseudospin formalism can be applied [5]. The dielectric properties and electron spectrum of this pseudospin-electron model were studied within the Hubbard-I approximation [6]. It was shown that the interaction of electrons with anharmonic 
vibrational modes leads to the existence of extra sub-bands and causes their additional narrowing; the electron contribution to the transverse dielectric susceptibility can be of significant magnitude resulting in high values of this quantity. The investigation performed within the mean field approximation [7] gave evidence for possibility of the phase transition into a charge-ordered state at electron concentration $n \sim 1$. It pointed to the fact that an interaction between electron subsystem and pseudospin one leads to the appearance of an effective $V n_{i} n_{j}$ type term. It is responsible for a phase transition with a modulation of site concentration. The results obtained in the general random phase approximation (GRPA) method [8] confirm it. Moreover, it was shown there were a phase transition in the centre of the Brillouin zone at low electron concentrations. This transition manifests itself in the divergency of susceptibility $\chi_{\perp}$ at a certain value of temperature.

In some experiments $\mathrm{YBaCuO}$ was found to be both pyroelectric and piezoelectric, implying existence of the macroscopic polarization directed along the $c$-axis [9]. In order to explain such type behaviour in the framework of pseudospin-electron model it is important to take into account the interaction between pseudospins. This interaction of Mitsui type can originate the ordering of pseudospins and appearance of a ferroelectric phase [10].

In this paper we investigate the dielectric and thermodynamical properties of the two-subblattice pseudospin-electron model. We shall restrict our consideration to the case of zero electron hopping. In Sec.2 we introduce the Hamiltonian of the model. In Sec.3 we calculate the transverse dielectric susceptibility in the noninteracting clusters approximation. The thermodynamical properties and susceptibility in the presence of pseudospinpseudospin interaction are considered within mean field interaction (MFA) in Sec.4. Finally, in Sec.5, we present a summary of results and conclusions.

\section{The Hamiltonian of two-sublattice model.}

The Hamiltonian of the model with lattice local anharmonicity written with the use of pseudospin formalism has the form

$$
\begin{aligned}
H & =H_{e}+H_{s}+H_{e-s}+H_{s-s}, \\
H_{e} & =-\mu \sum_{n, s}\left(n_{n 1}^{s}+n_{n 2}^{s}\right)+U \sum_{n}\left(n_{n 1}^{\uparrow} n_{n 1}^{\downarrow}+n_{n 2}^{\uparrow} n_{n 2}^{\downarrow}\right), \\
H_{s} & =-h \sum_{n}\left(S_{n 1}^{z}-S_{n 2}^{z}\right)-\Omega \sum_{n}\left(S_{n 1}^{x}+S_{n 2}^{x}\right), \\
H_{e-s} & =g \sum_{n, s}\left(n_{n 1}^{s} S_{n 1}^{z}-n_{n 2}^{s} S_{n 2}^{z}\right), \\
H_{s-s} & =-J \sum_{n} S_{n 1}^{z} S_{n 2}^{z}-\frac{1}{2} \sum_{n, n^{\prime}} \sum_{\alpha, \beta} J_{n n^{\prime}}^{\alpha \beta} S_{n \alpha}^{z} S_{n \beta}^{z} .
\end{aligned}
$$

Here, $n_{n \alpha}^{s}$ is the operator of the number of electrons with spin $s$ and $S_{n \alpha}^{z}$ stands for the operator of the pseudospin at the $n$ cell in $\alpha$ plane ( $\alpha=$ 1,2 in the two-sublattice case). $H_{e}$ is the Hubbard Hamiltonian without term describing transfer of electrons. $H_{s}$ is the pseudospin part of the Hamiltonian and $h$ describes the asymmetry of well potential, $\Omega$ is the tunnelling splitting of the vibrational mode. $H_{e-s}$ is the term describing the interaction between electrons and pseudospins. $H_{s-s}$ gives the interaction between pseudospins; the interaction $-J S_{n 1}^{z} S_{n 2}^{z}$ inside one cell clusters is 
separated. We consider two different variants of model realization: $\mu=$ const, $n=n(\mu)$ and $n=$ const, $\mu=\mu(n)$ with the purpose of elucidating the role of structural elements or electron bands which are not explicitly included into model. Hamiltonian (1) is invariant under transformation $n_{n \alpha}^{s} \rightarrow 1-n_{n \alpha}^{s}, h \rightarrow 2 g-h, \mu \rightarrow-\mu-U$. It allows us to use (1) for the description of hole-pseudospin system as well.

Let us introduce the single-cluster basis of states $\left|R_{i}\right\rangle \equiv\left|n_{i 1}^{\uparrow}, n_{i 1}^{\downarrow}, n_{i 2}^{\uparrow}, n_{i 2}^{\downarrow}\right\rangle$ $\oplus\left|S_{i 1}^{z}, S_{i 1}^{z}\right\rangle$, which consists of sixty-four state vectors

$$
\begin{aligned}
|1\rangle & =|0,0,0,0, \uparrow, \uparrow\rangle \\
|2\rangle & =|0,0,0,0, \uparrow, \downarrow\rangle \\
|3\rangle & =|0,0,0,0, \downarrow, \uparrow\rangle \\
|4\rangle & =|0,0,0,0, \downarrow, \downarrow\rangle \\
|5\rangle & =|0,0,0,1, \uparrow, \uparrow\rangle \\
& \cdots \\
|64\rangle & =|1,1,1,1, \downarrow, \downarrow\rangle .
\end{aligned}
$$

On this basis the Hamiltonian has a block diagonal form with size of blocks $4 \times 4$. Indeed, only the pseudospin part of Hamiltonian is non-diagonal because there is no interaction between electrons on different planes. The $i$ - th block, which corresponds to a fixed electron configuration $\left|K_{i}\right\rangle \equiv$ $\left|n_{i 1}^{\uparrow}, n_{i 1}^{\downarrow}, n_{i 2}^{\uparrow}, n_{i 2}^{\downarrow}\right\rangle$, has the following form

$$
\left(\begin{array}{ccccc}
\varepsilon_{e l}^{K_{i}}+\frac{g}{2}\left(n_{i 1}-n_{i 2}\right)-\frac{J}{4} & -\frac{\Omega}{2} & -\frac{\Omega}{2} & 0 & \left|K_{i}, \uparrow, \uparrow\right\rangle \\
-\frac{\Omega}{2} & \varepsilon_{e l}^{K_{i}}+\frac{g}{2}\left(n_{i 1}+n_{i 2}\right)+\frac{J}{4}-h & 0 & -\frac{\Omega}{2} & \left|K_{i}, \uparrow, \downarrow\right\rangle \\
-\frac{\Omega}{2} & 0 & \varepsilon_{e l}^{K_{i}}-\frac{g}{2}\left(n_{i 1}+n_{i 2}\right)+\frac{J}{4}+h & -\frac{\Omega}{2} & \left|K_{i}, \downarrow, \uparrow\right\rangle \\
0 & -\frac{\Omega}{2} & -\frac{\Omega}{2} & \varepsilon_{e l}^{K_{i}}-\frac{g}{2}\left(n_{i 1}-n_{i 2}\right)-\frac{J}{4}^{2} & \left|K_{i}, \downarrow, \downarrow\right\rangle .
\end{array}\right.
$$

Here $n_{i \alpha}$ is an eigenvalue of operator $\hat{n}_{i \alpha}^{\uparrow}+\hat{n}_{i \alpha}^{\downarrow}$, and $\varepsilon_{e l}^{K_{i}}$ is the electron contribution to the energy of the $\left|K_{i}\right\rangle$ state.

In the case $J=0$, the general problem can be reduced to the problem of two noninteracting single-site subsystems. Then we can use approach proposed in [6]. The eigenvalues of (1) may be obtained by orthogonal transformation

$$
H_{d}=A H A^{T},
$$

where matrix A has a block diagonal form similar to the form of matrix $H$. The block which is assigned to (3) can be obtained via

$$
A_{K_{i}}=\left(\begin{array}{cc}
\cos \varphi_{i 1} & \sin \varphi_{i 1} \\
-\sin \varphi_{i 1} & \cos \varphi_{i 1}
\end{array}\right) \otimes\left(\begin{array}{cc}
\cos \varphi_{i 2} & \sin \varphi_{i 2} \\
-\sin \varphi_{i 2} & \cos \varphi_{i 2}
\end{array}\right),
$$

where

$$
\cos 2 \varphi_{i \alpha}=\frac{n_{i \alpha} g-h}{\sqrt{\left(n_{i \alpha} g-h\right)^{2}+\Omega^{2}}} .
$$

The energy spectrum is

$$
\varepsilon_{K_{i}}=\varepsilon_{e l}^{K_{i}} \pm \sqrt{\left(n_{i 1} g-h\right)^{2}+\Omega^{2}} \pm \sqrt{\left(n_{i 2} g-h\right)^{2}+\Omega^{2}} .
$$


For example, when $|K\rangle=|0,1,1,1\rangle$,

$$
\begin{aligned}
& \varepsilon_{K_{i}}^{1}=-3 \mu+U+\sqrt{(g-h)^{2}+\Omega^{2}}+\sqrt{(2 g-h)^{2}+\Omega^{2}}, \\
& \varepsilon_{K_{i}}^{2}=-3 \mu+U+\sqrt{(g-h)^{2}+\Omega^{2}}-\sqrt{(2 g-h)^{2}+\Omega^{2}}, \\
& \varepsilon_{K_{i}}^{3}=-3 \mu+U-\sqrt{(g-h)^{2}+\Omega^{2}}+\sqrt{(2 g-h)^{2}+\Omega^{2}}, \\
& \varepsilon_{K_{i}}^{4}=-3 \mu+U-\sqrt{(g-h)^{2}+\Omega^{2}}-\sqrt{(2 g-h)^{2}+\Omega^{2}} .
\end{aligned}
$$

Both the spectrum of the model and matrix $A$ in the general case $(J \neq 0)$ have been determined but the results are not given here for the sake of brevity.

\section{Dielectric properties along $c$-axis in the absence of the pseudospin-pseudospin interaction.}

Let us consider the simplified case $J_{n n^{\prime}}^{\alpha \beta}=0$. Then Hamiltonian (1) describes the behaviour of a system of "noninteracting" clusters. The coupling between them exists only through the common reservoir of charge carriers (described by chemical potential $\mu$ ). The latter one is the same for both of sublattices. That is why the case $J=0$ is not reduced to the one sublattice case [8]. We included in the Hamiltonian a part which describes the influence of an electric field perpendicular to the planes:

$$
H_{i n t}=-E_{\perp} \sum_{n} P_{n}^{z}
$$

where $P_{n}^{z}$ is the polarization operator along the field direction. It is given by the formula

$$
P_{n}^{z}=d_{s}\left(S_{n 1}^{z}+S_{n 2}^{z}\right)+d_{e}\left(n_{n 1}-n_{n 2}\right),
$$

where $d_{s}$ is the dipole moment connected with pseudospin flipping (transition of oxygen atom from one minimum of double well potential to another); $d_{e}$ is the dipole moment of electron charge transfer between planes. Then the transverse dielectric susceptibility is given by

$$
\chi_{\perp}=\left.\frac{1}{v_{c} N} \frac{\partial\left\langle\sum_{n} P_{n}^{z}\right\rangle_{H+H_{i n t}}}{\partial E_{\perp}}\right|_{\mid E_{\perp}=0}=\left.\frac{1}{v_{c}} \frac{\partial\left\langle P^{z}\right\rangle_{H+H_{i n t}}}{\partial E_{\perp}}\right|_{\mid E_{\perp}=0},
$$

here $N$ is the total number of sites, $v_{c}$ is is the volume of a primitive cell. In numerical calculations we shall describe the susceptibility by the dimensionless quantity $\tilde{\chi}_{\perp}=\frac{g v_{c}}{d_{e}^{2}} \chi_{\perp}$.

In order to understand the possible temperature behaviour of $\chi_{\perp}$, firstly, we consider how the ground state of the system is changed at different values of model parameters. Fig.1 illustrates the ground state diagram for $\Omega=0$, $J>0, U \rightarrow \infty$ - the solid lines on this figure separate regions with different ground states. The chemical potential changes along the $\mathrm{AC}_{1} \mathrm{DE}$ segment at $n \sim 0$ and along $\mathrm{ABCE}$ at $n \sim 2$. One can draw a line parallel to the $h$-axis to consider the $\mu=$ const case. 


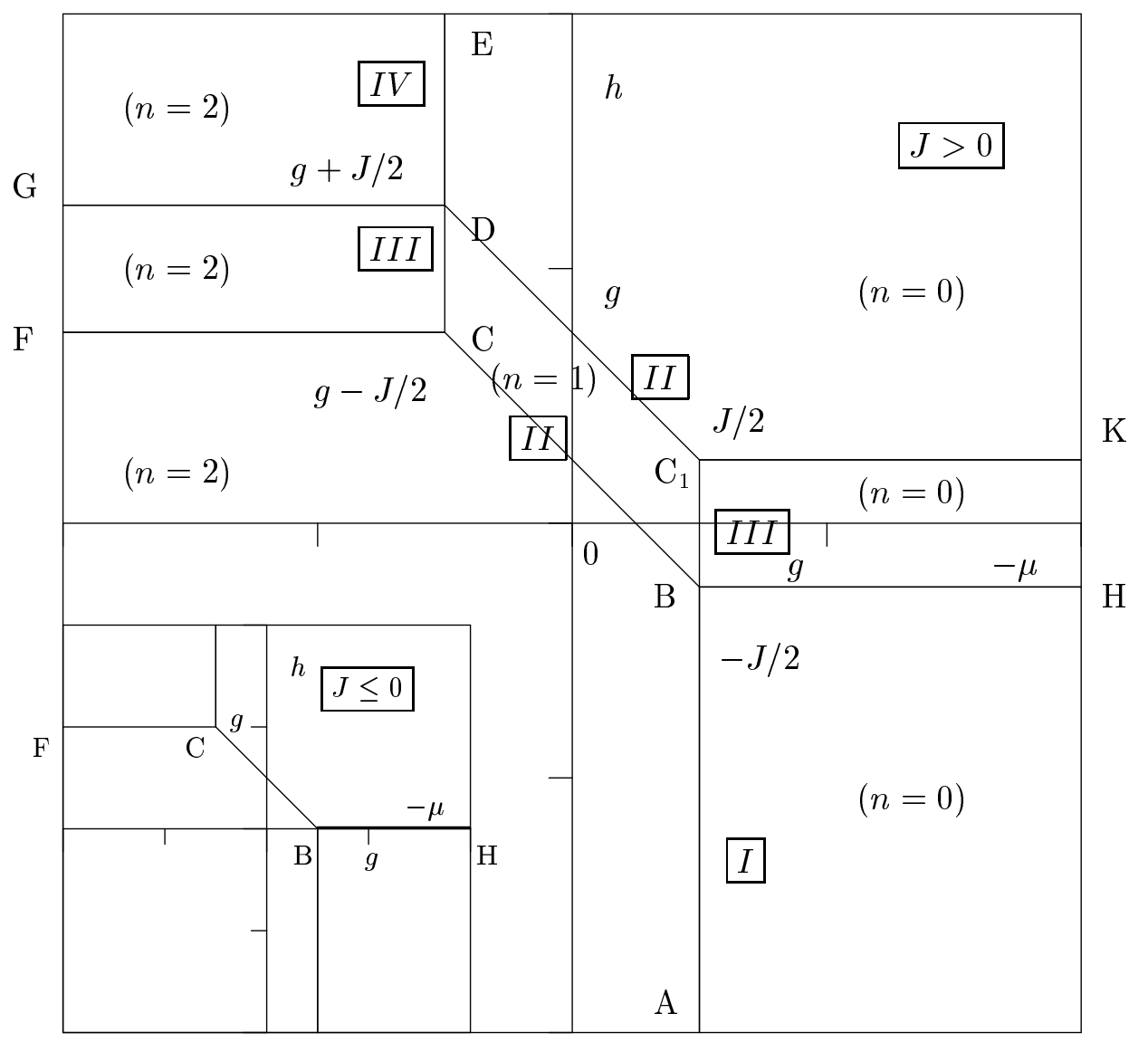

Figure 1. The ground state diagram. Average concentration of electrons is shown in the brackets. 
The calculations show that the eigenvalues of the polarization operator in the ground state have a nonzero values at $J>0$ only inside the region KHFG; its width is proportional to $J$ (at $J<0$ this region is absent). Inside region $\mathrm{BC}_{1} \mathrm{KH}$ the ground state is doubly degenerate and is described by the state vectors $|0,0,0,0, \uparrow, \uparrow\rangle,|0,0,0,0, \downarrow, \downarrow\rangle$. In FGDC region states with the lowest energy $\left|K_{i}, \uparrow, \uparrow\right\rangle$ and $\left|K_{i}, \downarrow, \downarrow\right\rangle$; in region $\mathrm{BCDC}_{1}$ the ground state is four-fold degenerate. The electron part of the ground state dipole moment is different from zero only inside the region $\mathrm{BCDC}_{1}$. The susceptibility $\chi_{\perp}$ is proportional to $\mathrm{e}^{-\alpha T}(\alpha>0)$ outside of those regions and it is obeyed the $\frac{1}{T}$ law inside of them:

$$
\begin{aligned}
& n=\text { const } \\
& I, I V \quad \chi_{\perp}=\frac{\beta}{v_{c}} \frac{(2-n) n}{2} d_{e}^{2} ; \\
& \text { II } \quad \chi_{\perp}(0<n<1)=\frac{\beta}{v_{c}} n\left(d_{s}-d_{e}\right)^{2}, \\
& \chi_{\perp}(1<n<2)=\frac{\beta}{v_{c}}(2-n)\left(d_{s}-d_{e}\right)^{2} \text {; } \\
& \text { III } \quad \chi_{\perp}(0<n<1)=\frac{\beta}{v_{c}}\left\{n\left(d_{s}-d_{e}\right)^{2}+(1-n) d_{s}^{2}\right\}, \\
& \chi_{\perp}(1<n<2)=\frac{\beta}{v_{c}}\left\{(2-n)\left(d_{s}-d_{e}\right)^{2}+(n-1) d_{s}^{2}\right\} \text {. } \\
& \mu=\text { const } \\
& \mu \gg g \quad \chi_{\perp}=\frac{\beta d_{s}^{2}}{v_{c}\left(1+e^{-\beta \frac{J}{2}} \operatorname{ch} \beta(g-h)\right)} ; \\
& |\mu| \gg g, \mu<0 \quad \chi_{\perp}=\frac{\beta d_{s}^{2}}{v_{c}\left(1+e^{-\beta \frac{J}{2}} \operatorname{ch} \beta h\right)} ; \\
& \mu \sim 0 \quad \chi_{\perp}(-h \gg g)=\frac{\beta}{v_{c}} d_{e}^{2} e^{-\beta \frac{g}{2}} \\
& \chi_{\perp}(h \gg g)=4 \frac{\beta}{v_{c}} d_{e}^{2} e^{-\beta \frac{g}{2}}, \\
& \chi_{\perp}\left(h \sim \frac{g}{2}\right)=\frac{\beta}{v_{c}}\left(d_{s}-d_{e}\right)^{2} .
\end{aligned}
$$

The formulae (11), (12) represent the main dependencies in the limit of low temperatures and are obtained in the case of infinite Coulomb repulsion $U$.

Figs.2,3 display the behaviour of the susceptibility $\chi_{\perp}$ with the change of the asymmetry parameter $h$. Essential changes of $\chi_{\perp}$ take place in the interval corresponding to the region $\mathrm{BCDC}_{1}$. The total susceptibility is proportional here to $\left(d_{s}-d_{e}\right)^{2}$. It explains at $d_{s}>0$ and $d_{e}>0$ the sharp fall of $\chi_{\perp}$ in the $n=$ const case.

The influence of tunnelling motion in anharmonic potential wells on the susceptibility $\chi_{\perp}$ is essential when $\Omega$ splits the states which make at low temperatures the main contribution to the polarization. This effect can be taken into account by the perturbation theory. In this case the temperature dependence of $\chi_{\perp}$ is transformed from Curie's law to a more complicated behaviour. The perturbational approach gives the following results: 


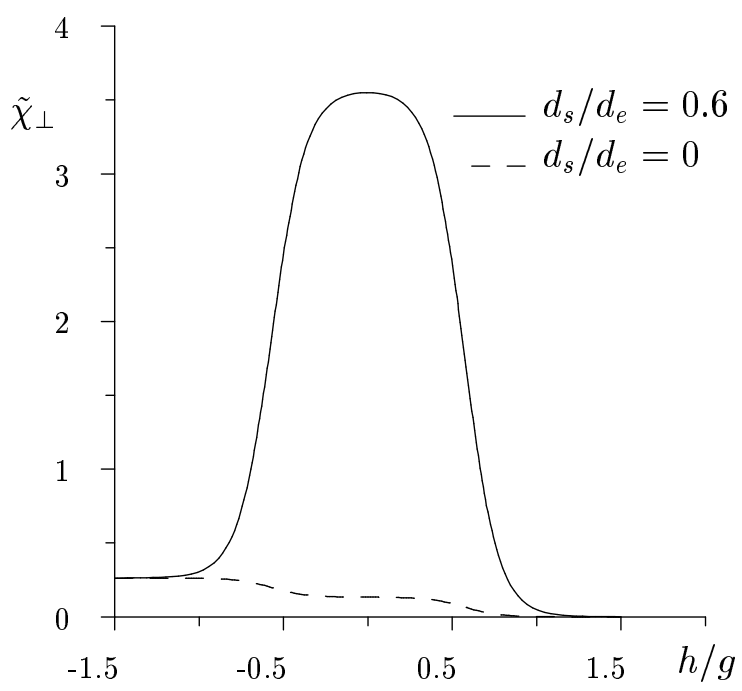

Figure 2. The susceptibility $\tilde{\chi}_{\perp}$ as function of asymmetry parameter in the regime $\mu=$ const. $(J / g=-1, \Omega=0, T / g=0.1, \mu / g=-1)$.

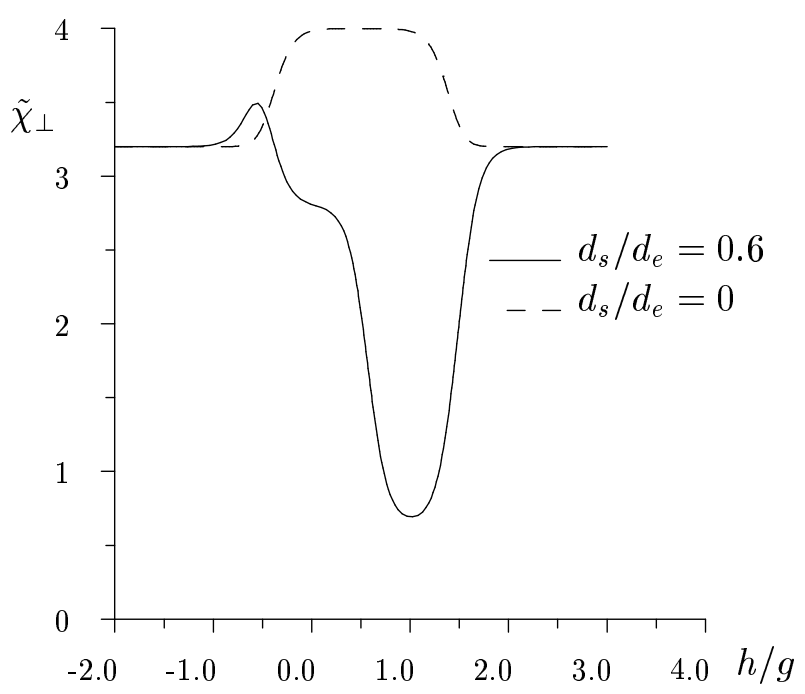

Figure 3 . The susceptibility $\tilde{\chi}_{\perp}$ as function of asymmetry parameter in the regime $n=$ const. $(J / g=-1, \Omega=0, T / g=0.1, n=0.4)$. 


$$
\begin{aligned}
& n=\text { const } \\
& I, I V \quad \chi_{\perp}=\frac{\beta}{v_{c}} \frac{(2-n) n}{2} d_{e}^{2} ; \\
& I I, I I I \quad \chi_{\perp}(0<n<1)=\frac{\beta}{v_{c}} n\left(d_{s}-d_{e}\right)^{2}, \\
& \chi_{\perp}(1<n<2)=\frac{\beta}{v_{c}}(2-n)\left(d_{s}-d_{e}\right)^{2} \text {; } \\
& \mu=\text { const } \\
& \mu \gg g \quad \chi_{\perp}=\frac{d_{s}^{2}}{v_{c}\left|\zeta_{1}+\zeta_{2}\right|} \times \frac{\operatorname{sh} \beta\left|\zeta_{1}+\zeta_{2}\right|}{\operatorname{ch} \beta\left(\zeta_{1}+\zeta_{2}\right)+e^{-\beta \frac{J}{2}} \operatorname{ch} \beta\left(g-h+\zeta_{1}-\zeta_{2}\right)}, \\
& \text { where } \zeta_{1}=\frac{\Omega^{2} / 4}{\frac{J}{2}+g-h}, \zeta_{2}=\frac{\Omega^{2} / 4}{\frac{J}{2}-g+h} ; \\
& -\mu \gg g \quad \chi_{\perp}=\frac{d_{s}^{2}}{v_{c}\left|\eta_{1}+\eta_{2}\right|} \times \frac{\operatorname{sh} \beta\left|\eta_{1}+\eta_{2}\right|}{\operatorname{ch} \beta\left(\eta_{1}+\eta_{2}\right)+e^{-\beta \frac{J}{2}} \operatorname{ch} \beta\left(h+\eta_{1}-\eta_{2}\right)}, \\
& \text { where } \eta_{1}=\frac{\Omega^{2} / 4}{\frac{J}{2}+h}, \eta_{2}=\frac{\Omega^{2} / 4}{\frac{J}{2}-h} \\
& \mu \sim 0 \quad \chi_{\perp}(-h \gg g)=\frac{\beta}{v_{c}} d_{e}^{2} e^{-\beta \frac{g}{2}}, \\
& \chi_{\perp}(h \gg g)=4 \frac{\beta}{v_{c}} d_{e}^{2} e^{-\beta \frac{g}{2}}, \\
& \chi_{\perp}\left(h \sim \frac{g}{2}\right)=\frac{\beta}{v_{c}}\left(d_{s}-d_{e}\right)^{2} .
\end{aligned}
$$

The tunnelling lowers the value of the transverse dielectric susceptibility $\chi_{\perp}$ almost in all range of parameters. When $\mu \rightarrow \pm \infty$ (the case when the influence of electron subsystem is absent), the susceptibility achieves a maximum at low temperatures (formula (14)) and in the case of degenerate ground state $\chi_{\perp} \stackrel{T \rightarrow 0}{\sim} \frac{d_{s}^{2}}{\Omega^{2}}$. In the region $\mathrm{BCDC}_{1}$ the character of temperature dependence of the susceptibility is not changed. Here the tunnelling splitting only shifts the states which contribute to the polarizability.

\section{Thermodynamics of the system in the mean field ap- proximation.}

The pseudospin part of Hamiltonian $H_{s}+H_{s-s}$ apart from the term containing $J$ interaction corresponds to the Hamiltonian of Mitsui model. This model is used for the explanation of phase transitions in some kinds of ferroelectrics with hydrogen bonds [11]. It was also proposed for the description of $\mathrm{YBaCuO}$-type superconductor properties [10]. The inclusion of pseudospin-pseudospin interaction can lead to the appearance in the model of a phase with pseudospin ordering, characterizing by nonzero total dipole 
moment. This interaction can be considered within the MFA:

$$
\begin{aligned}
H_{s-s}^{\prime} & =-\frac{1}{2} \sum_{n, n^{\prime}} \sum_{\alpha, \beta} J_{n n^{\prime}}^{\alpha \beta} S_{n \alpha}^{z} S_{n \beta}^{z} \rightarrow \\
& \rightarrow-\sum_{n, n^{\prime}} \sum_{\alpha, \beta} J_{n n^{\prime}}^{\alpha \beta}\left\langle S_{\alpha}^{z}\right\rangle S_{n \beta}^{z}+\frac{1}{2} \sum_{n, n^{\prime}} \sum_{\alpha, \beta} J_{n n^{\prime}}^{\alpha \beta}\left\langle S_{\alpha}^{z}\right\rangle\left\langle S_{\beta}^{z}\right\rangle .
\end{aligned}
$$

We shall consider as above the limit of infinite large Coulomb repulsion $U$ and neglect for the sake of simplicity tunnelling splitting of energy levels.

Let us introduce variables $\eta=\left\langle S_{1}^{z}+S_{2}^{z}\right\rangle$ (the order parameter) and $\xi=\left\langle S_{1}^{z}-S_{2}^{z}\right\rangle$. In the regime $\mu=$ const we obtain equations for $\eta$ and $\xi$ from the condition of thermodynamical potential $\Omega_{M F}$ minimum

$$
\begin{gathered}
\left\{\begin{array}{c}
\left(\frac{\partial \Omega_{M F}}{\partial \eta}\right)_{\mu}=0 ; \\
\left(\frac{\partial \Omega_{M F}}{\partial \xi}\right)_{\mu}=0 .
\end{array}\right. \\
\Omega_{M F}=-\frac{T}{N} \ln \operatorname{sp}\left(\mathrm{e}^{-\beta\left(H_{M F}-\mu \hat{n}\right)}\right), \\
\Omega_{M F}=\frac{1}{4}\left\{\left(J_{11}+J_{12}\right) \eta^{2}+\left(J_{11}-J_{12}\right) \xi^{2}\right\}- \\
-T \ln \left[2\left\{e^{\beta \frac{J}{4}} \operatorname{ch} \beta \frac{\left(J_{11}+J_{12}\right) \eta}{2}+e^{-\beta \frac{J}{4}} \operatorname{ch} \beta\left(h+\frac{\left(J_{11}-J_{12}\right) \xi}{2}\right)\right\}+\right. \\
+8 e^{\beta \mu}\left\{e^{\beta \frac{J}{4}} \operatorname{ch} \beta \frac{\left(J_{11}+J_{12}\right) \eta}{2} \operatorname{ch} \beta \frac{g}{2}+e^{-\beta \frac{J}{4}} \operatorname{ch} \beta\left(h+\frac{\left(J_{11}-J_{12}\right) \xi}{2}-\frac{g}{2}\right)\right\} \\
\left.+8 e^{2 \beta \mu}\left\{e^{\beta \frac{J}{4}} \operatorname{ch} \beta \frac{\left(J_{11}+J_{12}\right) \eta}{2}+e^{-\beta \frac{J}{4}} \operatorname{ch} \beta\left(h+\frac{\left(J_{11}-J_{12}\right) \xi}{2}-g\right)\right\}\right],
\end{gathered}
$$

where $N$ is the total number of cells and $J_{11}=\sum_{n^{\prime}} J_{n n^{\prime}}^{11}, J_{12}=\sum_{n^{\prime}} J_{n n^{\prime}}^{12}$.

For the case $n=$ const we should use the condition of the minimum of free energy $F_{M F}=\Omega_{M F}+\mu n$ and supplement the system (16) with the equation for chemical potential $\mu(n)$

$$
\left\langle\hat{n} \mathrm{e}^{-\beta\left(H_{M F}\right)}\right\rangle=n .
$$

When the system of equations $(16,19)$ has non-zero solutions for $\eta$ and the corresponding thermodynamic potential has a minimum, then our system is in the ferroelectric phase.

Obtained phase diagrams for cases $\mu=$ const and $n=$ const are shown in the Figs.4-6 (all the parameters are normalized by $J_{11}+J_{12}>0$ ). One can see that at $\frac{J_{11}-J_{12}}{J_{11}+J_{12}}=-1$ (there exists only pseudospin-pseudospin interaction between planes) only a phase transition of second order into ferroelectric phase takes place. One can find its temperature using Landau theory of second order type phase transitions.

The increase of the parameter $\frac{J_{11}-J_{12}}{J_{11}+J_{12}}$ leads to narrowing of the ferroelectric region and appearance of a first order type phase transition. In the regions with degenerate ground state the ferroelectric phase is always present. The long-range interaction splits ground states $\left|K_{i}, \uparrow, \uparrow\right\rangle$ as well as $\left|K_{i}^{\prime}, \downarrow, \downarrow\right\rangle$ and polarizes the system. 

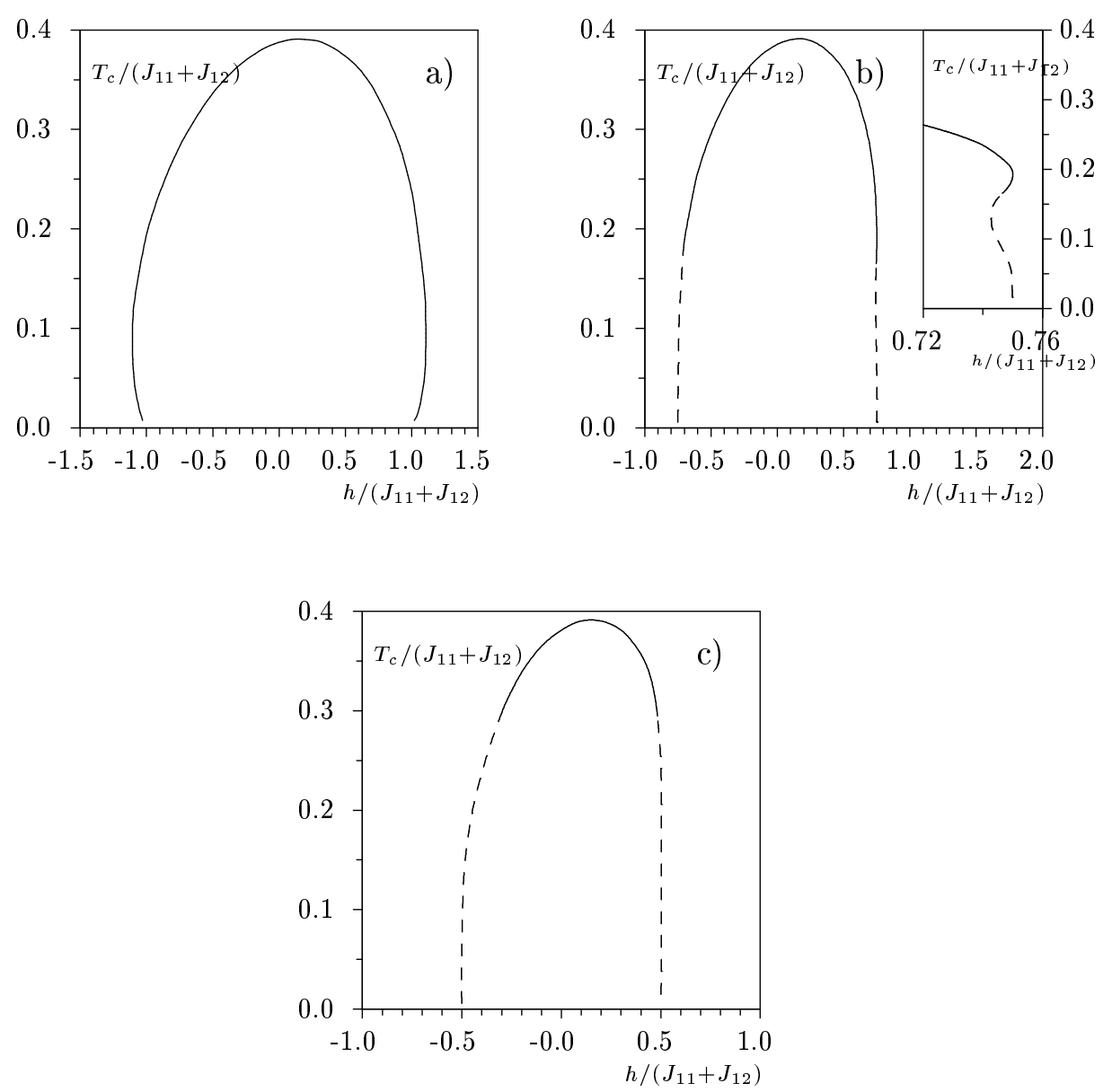

Figure 4. Dependence of the temperature of ferroelectric phase transition $T_{c}$ on $h$ at different values of parameter $J_{11}-J_{12}$ in the regime $\mu=$ const: a) $\frac{J_{11}-J_{12}}{J_{11}+J_{12}}=-1$, b) $\frac{J_{11}-J_{12}}{J_{11}+J_{12}}=0$, c) $\frac{J_{11}-J_{12}}{J_{11}+J_{12}}=1$. Other parameters: $J /\left(J_{11}+\right.$ $\left.J_{12}\right)=-1, g /\left(J_{11}+J_{12}\right)=1, \mu /\left(J_{11}+J_{12}\right)=-1$. The phase transitions can be of second (solid lines) or of first order (dotted lines). 

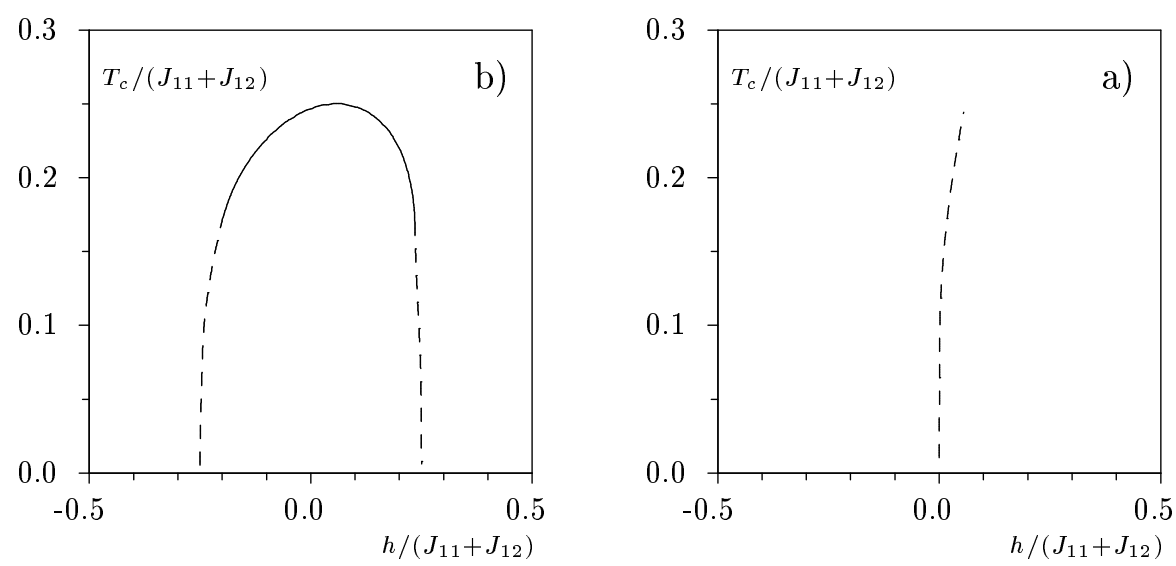

Figure 5. Dependence of the temperature of ferroelectric phase transition $T_{c}$ on $h$ at different values of parameter $J_{11}-J_{12}$ in the regime $\mu=$ const: a)

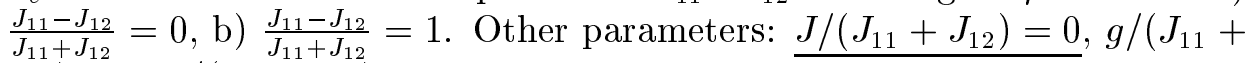
$\left.J_{12}\right)=1, \mu /\left(J_{11}+J_{12}\right)=-1$. The width of ferroelectric phase region in the case b) is equal to zero.

The width of the ferroelectric phase region is proportional to $J$ in the $\mu=$ const regime. It points of explicit taking account the interaction between pseudospins inside the cluster to be important. Let us set $J=0$. Then in the limit $\mu \rightarrow-\infty, J_{12}=0$ our model transforms into Ising model in the transverse field. Here the phase transition with zero value of order parameter and a sharp change of parameter $\xi=\left\langle S_{1}-S_{2}\right\rangle$ takes place (see Fig.5 b). This result is valid also at finite values of chemical potential because as the Fig.1 indicates, the regions with degenerate ground state are absent at $J=0$.

In the $n=$ const regime the interval of $h$ values at which the ferroelectric phase exists at low concentrations corresponds to the segment $\mathrm{BC}_{1} \mathrm{D}$ on Fig.1 (or to the segment BCD at $n \sim 2$ ). When the interaction between pseudospins inside clusters and long-range interaction $J_{12}$ are equal to zero, the ferroelectric phase persists and the effective size of interval of corresponding values of $h$ parameter is proportional to the length of segment BC.

The presence of a phase transitions manifests in the peculiar dependencies on $h$ of dielectric susceptibility (Figs.7,8). In the vicinity of a second order phase transition point the susceptibility increases drastically. At the point of first order phase transition the susceptibility jumps to another value without singularities.

The long-range interaction eliminates the $\frac{1}{T}$ law. It may be shown easy in the $\mu=$ const regime. We can neglect the field dependence of parameters $\eta, \xi(\eta=1$ and $\xi=0$ in ferroelectric phase) and use the formula

$$
\chi_{\perp}=\frac{\beta}{v_{c}}\left\langle\left(P^{z}\right)^{2}\right\rangle-\frac{\beta}{v_{c}}\left\langle P^{z}\right\rangle^{2} .
$$

$\left\langle P^{z}\right\rangle$ is different from zero in the ferroelectric phase. Moreover, $\left\langle P^{z^{2}}\right\rangle^{T \rightarrow 0}=$ 

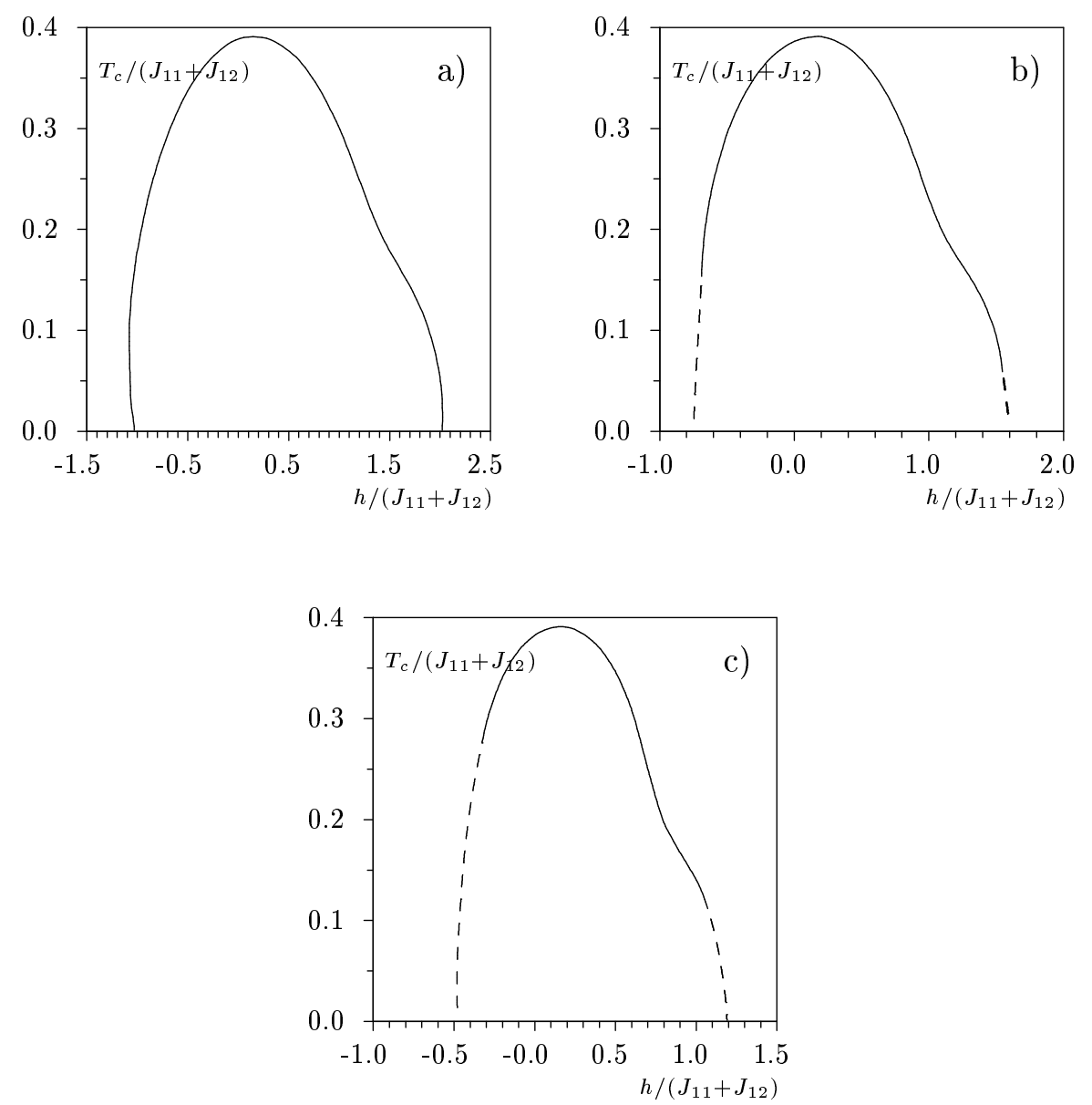

Figure $6 . h$ dependence of the temperature of ferroelectric phase transition $T_{c}$ at different values of parameter $J_{11}-J_{12}$ in the regime $n=$ const: a) $\frac{J_{11}-J_{12}}{J_{11}+J_{12}}=-1$, b) $\frac{J_{11}-J_{12}}{J_{11}+J_{12}}=0$, c) $\frac{J_{11}-J_{12}}{J_{11}+J_{12}}=1$. Other parameters: $J /\left(J_{11}+\right.$ $\left.J_{12}\right)=-1, g /\left(J_{11}+J_{12}\right)=1 n=0.4$. Solid lines and dotted lines represent second order and first order phase transitions respectively. 

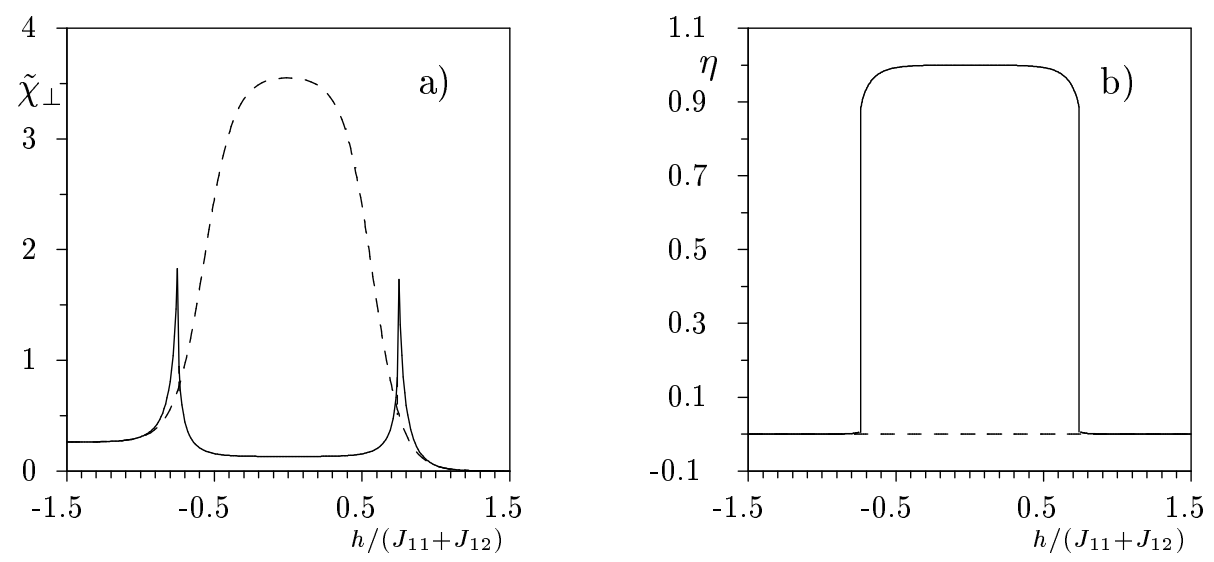

Figure 7. Transverse dielectric susceptibility and order parameter vs. $h /\left(J_{11}+J_{12}\right)$ in the regime $\mu=$ const. The first order phase transition takes place. $\frac{\mu}{J_{11}+J_{12}}=-1, \frac{J}{J_{11}+J_{12}}=-1, \frac{g}{J_{11}+J_{12}}=1, \frac{T}{J_{11}+J_{12}}=0.1, J_{11}-J_{12}=0$, $d_{s} / d_{e}=0.6$; dotted line presents result for $J_{n n^{\prime}}^{\alpha \beta} \rightarrow 0$.

$\left\langle P^{z}\right\rangle^{2}$. In the limit $\mu \rightarrow-\infty$, for example,

$$
\chi_{\perp}=4 \beta d_{s}^{2} e^{-\beta\left|J_{11}+J_{12}\right|}
$$

in the ferroelectric phase $\left(|h|<\frac{J}{2}, J>0\right)$. The temperature behaviour of susceptibility is changed also in the paraphase $\left(|h|>\frac{J}{2}, J>0\right)$ :

$$
\chi_{\perp}=\frac{\beta d_{s}^{2}}{1+e^{-\beta \frac{J}{2}} \operatorname{ch} \beta\left(\frac{J_{11-J_{12}}}{2}+|h|\right)} .
$$

The last expression at $J_{11}=J_{12}=0$ agrees with the result obtained in the case of the absence of a long-range interaction (12).

\section{Conclusions.}

In the framework of two-sublattice pseudospin-electron model the transverse dielectric susceptibility $\chi_{\perp}$ is calculated in the case of the absence of electron transfer. It is shown that if $J>0$ ( $\mathrm{J}$ describes the interaction between pseudospins in the same cell), there is a region of values of $h$ parameter with width $\sim J$ where the ground state of the system is degenerate. Here, in the regime $\mu=$ const, susceptibility is inversely proportional at low temperatures to the temperature. By contrast, in the regime $n=$ const $(\mu=\mu(n))$ the dielectric susceptibility obeys Curie's law at all values of the model parameters. The role of tunnelling is significant in the region where $\Omega$ splits the degenerate ground state. Then the susceptibility achieves its maximum at $T=0\left(\chi_{\perp} \sim \frac{1}{\Omega^{2}}\right)$.

In the second part of this work the interaction between pseudospins was considered within the mean field approximation. In substance, we dealt with a Mitsui model supplemented by an interaction with an electron system. On the basis of this model the ferroelectric type instabilities and phase transitions, connected with macroscopic polarization along $c$-axis in the systems 

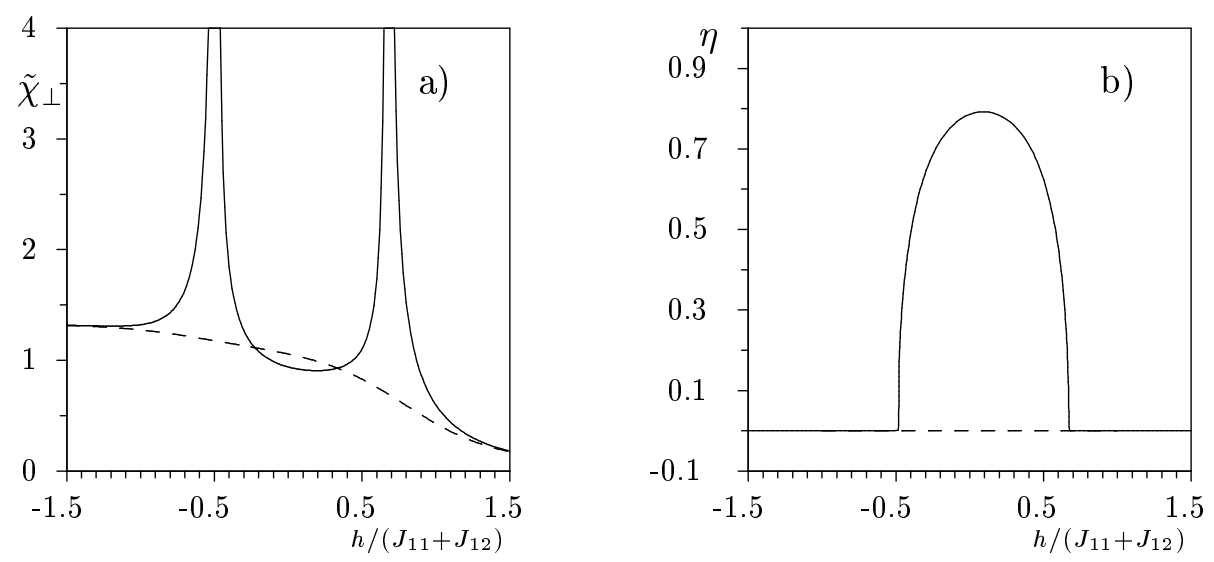

Figure 8. Transverse dielectric susceptibility and order parameter vs. $h /\left(J_{11}+J_{12}\right)$ in the regime $\mu=$ const. The second order phase transition takes place. $\frac{\mu}{J_{11}+J_{12}}=-1, \frac{J}{J_{11}+J_{12}}=-1, \frac{g}{J_{11}+J_{12}}=1, \frac{T}{J_{11}+J_{12}}=0.3$, $J_{11}-J_{12}=0, d_{s} / d_{e}=0.6$; dotted line presents result for $J_{n n^{\prime}}^{\alpha \beta} \rightarrow 0$.

like $\mathrm{YBaCuO}$ are considered. The interaction between electron and pseudospin subsystems leads to a shift of the ferroelectric phase compared to the pure Mitsui model and to a change in shape of the phase diagrams. The ferroelectric phase is always present in the region with degenerate ground state. The width of the corresponding interval of values of $h$ parameter is proportional to the constant of interaction between pseudospins inside cluster. The appearance of a ferroelectric phase changes the temperature behaviour of $\chi_{\perp}$ near phase transition points and eliminates Curie's law in the ferroelectric phase.

It should be mentioned that ferroelectric ordering of pseudospins has an effect on the redistribution of electrons (holes) between superconducting planes. On the other hand, electron transfer in the superconducting planes produces an effective interaction between pseudospins $[7,8]$. That is why it is necessary to consider both processes to describe the phase transitions and instabilities in real systems.

This work was supported, in part, by State Committee for Science and Technologies of Ukraine (grant No 09.01.01./009-94) and by the International Soros Science Education Program through grant No SPU 062062.

\section{References}

[1] Maruyama H., Ishii T., Bamba N., Maeda H., Koizumi A., Yoshikawa Y., Yamazaki $\mathrm{H}$. Temperature dependence of the EXAFS spectrum in $\mathrm{YBa}_{2} \mathrm{Cu}_{3} \mathrm{O}_{7-\delta}$ compounds. // Physica C, 1989, vol. 60, No 5/6, p. 524.

[2] Mustre de Leon J., Conradson S.D., Batistic' I., Bishop A.R., Raistrick I.D., Aronson M.C., Garzon F.H. Axial oxygen-centered lattice instabilities in $\mathrm{YBa}_{2} \mathrm{Cu}_{3} \mathrm{O}_{7}$ : An application of the analysis of extended x-ray-absorbtion fine structure in anharmonic systems. // Phys. Rev. B, 1992, vol. 45, p. 2447-2457.

[3] Ruani G., Taliani C., Muccini M., Conder K., Kaldis E., Keller H., Zech D., Muller K.A. Apex anharmonicity observed by Raman scattering in ${ }^{18} \mathrm{O}$ substituted $\mathrm{YBa}_{2} \mathrm{Cu}_{3} \mathrm{O}_{6+x}$. // Physica C, 1994, vol. 226, p. 101-105.

[4] Conradson S.D., Raistrick I.D. The axial oxygen atom and superconductivity in $\mathrm{YBa}_{2} \mathrm{Cu}_{3} \mathrm{O}_{7}$. // Science, 1989, vol. 243, No 4896, p. 1340. 
[5] Müller K.A. // Phase transitions, 1988 (Special issue).

[6] Stasyuk I.V., Shvaika A.M. Dielectric properties and electron spectrum of the Müller model in the HTSC theory. // Acta Physica Polonica A, 1993, vol. 84, No 2, p. 293-313.

[7] Stasyuk I.V., Danyliv O.D. The account of effective interaction in the Hubbard model with local anharmonicity. // Lviv, 1994, 24 p. (Preprint / Ukr. Acad. Sci. Inst. Cond. Matt. Phys.; ICMP-94-6U), (in Ukrainian).

[8] Stasyuk I.V., Shvaika A.M., Danyliv O.D. Dielectric instability and charge ordering in the local anharmonic model of high $T_{c}$ superconductors. // Molecular Physics Reports, 1995, vol. 9, p. 61-75.

[9] Mihailovic D., Heeger A.J. Pyroelectric and piezoelectric effects in single crystals of $\mathrm{YBa}_{2} \mathrm{Cu}_{3} \mathrm{O}_{7-\delta}$. // Solid State Comm., 1990, vol. 75, p. 319-323.

[10] Galbaatar T., Plakida N.M. Ferroelectric ordering induced by apical oxygen anharmonicity in $\mathrm{YBa}_{2} \mathrm{Cu}_{3} \mathrm{O}_{7}$. In: Intern. Conference $\mathrm{M}^{2} \mathrm{~S}-\mathrm{HTSC} \mathrm{IV}, 5-9$ July, 1994, Grenoble.

[11] Blinc R., Zeks B. Soft modes in ferroelectrics and antiferroelectrics. Mir, Moscow, 1975 (in Russian).

\title{
АНАЛІЗ НЕСТАБІЛЬНОСТЕЙ СЕГНЕТОЕЛЕКТРИЧНОГО ТИПУ У ДВОПІДГРАТКОВІЙ МОДЕЛІ СИСТЕМ З ВИСОКОТЕМПЕРАТУРНОЮ НАДПРОВІДНІСТЮ
}

\author{
О.Д.Данилів, І.В.Стасюк
}

\begin{abstract}
Проводиться дослідження двопідграткової псевдоспінової моделі типу Міцуї, яка доповнена взаємодією з електронною підсистемою. Такого типу модель може бути використана для опису діелектричних властивостей у надпровідників типу $\mathrm{YBaCuO}$ вздовж осі $c$ (псевдоспіни представляють ангармонічні коливання вершинних киснів О4). Поведінка поперечної діелектричної сприйнятливості системи $\chi_{\perp}$ розглянена в наближенні невзаємодіючих кластерів. Встановлено можливості різних температурних залежностей $\chi_{\perp}$.

В наближенні середнього поля розрахована вільна енергія моделі. Встановлено, що далекодіюча взаємодія між ангармонічними коливаннями кисню може сприяти порушенню симетрії та появі сегнетоелектричної компоненти псевдоспінового впорядкування, причому фазовий перехід в стан 3 нескомпенсованим дипольним моментом може бути як першого так $\mathrm{i}$ другого роду. Отримані відповідні $\left(T_{c}, h\right)$ діаграми (де $h$ параметер асиметрії поля, що діє на псевдоспіни) при різних значеннях констант далекодіючої взаємодії в режимах 3 фіксованим значенням електронної концентрації $(n=c o n s t)$ чи хімічного потенціалу $(\mu=$ const). Досліджена аномальна поведінка сприйнятливості $\chi_{\perp}$ поблизу фазових переходів.
\end{abstract}

